\title{
Fairness and Inequality in Power System Reliability: Summarizing Indices
}

\author{
Evelyn Heylen, Marten Ovaere ${ }^{\bullet}$, Stef Proost $\bullet$, Geert Deconinck, Dirk Van Hertem \\ KU Leuven, Department of Electrical Engineering, EnergyVille \\ Kasteelpark Arenberg 10 PB 2445, 3001 Leuven-Heverlee \\ - $K U$ Leuven, Faculty of Economics and Business, \\ Naamsestraat 69 PB 3500, 3000 Leuven
}

\begin{abstract}
As the power system is undergoing major changes that affect different stakeholders unequally, power system literature increasingly recognizes the importance of fairness. This paper focuses specifically on the concept and quantification of fairness in the context of power system reliability. Power system decisions not only affect the overall, system reliability level, but also the reliability level for individual end-users, such as generators, flexibility providers and end-consumers, depending on their location and characteristics. We analyze and propose Gini-based and variance-based fairness indices. The proposed indices summarize the inequality and inequity of the distribution of reliability between different entities in power systems in a single number, which is a measure of the perceived fairness of the reliability level. These indices allow decision makers to assess the effect of power system decisions on fairness of reliability, to track it over time and to take appropriate actions to decrease unfairness. This will help to ensure the social acceptability of power system decisions. The use of the fairness indices is illustrated using two case studies: real reliability data and system development.

Keywords: Fairness, Gini coefficient, Inequality, Inequity, Power system reliability
\end{abstract}

\section{Introduction}

Social acceptability is one of the aspects determining the feasibility of power system actions, such as building new lines, the installation of power flow control equipment, generation adequacy load-shedding [1], the application of new reliability criteria [2, 3], replacement and maintenance of infrastructure

${ }_{5}$ [4, 5], cross-border cooperation on balancing 6], etc. In a power system reliability context, social acceptability is determined by how the reliability level is perceived by the end-users. This depends on the one hand on the absolute level of reliability and on the other hand on how the positive and negative

* Corresponding author

Email address: evelyn.heylen@esat.kuleuven.be (Evelyn Heylen) 
consequences of a decision are distributed among the end-users, i.e., the fairness of the distribution [7] 1

The importance of fairness is increasingly recognized in the power system literature. Wolsink 9] found that equity and fairness are the main reasons for public opposition to wind farms, while Perlaviciute et al. [7] argued that the different drivers for public acceptability, of which fairness is one, should be assessed from the start of a project and during the implementation phase. This is also stated by Cohen et al. [10] in the context of local opposition to new transmission lines and by Groppi et al. [11] who stressed the importance of public engagement in network expansion. Also Wolf et al. [12] suggested that measures should be undertaken to raise public acceptance of transmission investments. Karimi et al. recognized the importance of an equitable network charging scheme [13]. The above studies indicate the need for adequate assessments of fairness in a power system context.

Nevertheless, the assessment of fairness of the distribution of reliability in power systems is rarely dealt with in existing literature. To our knowledge, only two papers touch upon the assessment of fairness in the context of power system reliability. First, Strbac et al. [14] recognize the importance of fairness, which they assess by studying detailed reliability data on graphs and in tables. Second, Latif et al. [15] take this one step further in the context of active power curtailment of rooftop photovoltaic (PV) systems. They define two measures that quantify fairness: the standard deviation of the absolute amount of PV energy curtailment and the standard deviation of PV energy curtailment normalized to the rated power of the PV installation. The variance and standard deviation are popular indices to measure dispersion in several application contexts and are also applied to quantify unfairness. Economic literature, however, has shown that alternative indices, such as the coefficient of variation and the Gini index, provide a better assessment of fairness. At present, these indices have not been applied in the power system context.

To improve the assessment of fairness of the distribution of reliability among end-users in power systems, we apply economic tools in the power system reliability context. First, we propose fairness ratios to assess the distribution of reliability among end-users based on two interpretations of fairness: equality and equity. Second, we develop and analyze Gini-based and variance-based fairness indices that summarize fairness of the distribution of reliability in a single value. The proposed fairness indices are generic, as they can be formulated in terms of various reliability indicators, such as energy not supplied, interruption cost, interruption duration, etc. The application and usefulness of the fairness indices are illustrated in two case studies where the reliability level for consumers is unequally affected.

\footnotetext{
${ }^{1}$ Nowadays, regulatory practice focuses on the absolute, average level of reliability in terms of indicators, such as system average interruption frequency index (SAIFI), system average interruption duration index (SAIDI), expected energy not supplied (EENS), interruption duration (ID), and interruption cost 8 .
} 
The first case study evaluates the fairness of the distribution of reliability among consumers in Norway between 2006 and 2016 based on real Norwegian reliability data. The regulator can execute this type of assessment to evaluate system operation in practice. The second case study illustrates how the fairness indices can be applied in system development to assess investment options and how system stakeholders can collect detailed information about the fairness using the proposed indices.

In general, the fairness indices can be applied to assess the impact of actions in system design and operation on the distribution of reliability over end-users. Besides building new lines, these actions comprise installing power flow control equipment, designing generation adequacy load-shedding plans, executing load curtailment actions and applying new reliability criteria. The generic design of the indices enables the assessment of the unfairness of reliability provided to different types of end-users, not only end-consumers, but also generators and flexibility providers, i.e., how often they cannot provide their service to their customers due to network issues. ${ }^{2}$

The remainder of this paper is organized as follows. Section 2 defines fairness in a power system reliability context in a qualitative way and introduces the fairness ratios. Section 3 discusses the developed Gini-based fairness indices and their strengths and weaknesses. Section 4 discusses the characteristics of currently used variance-based indices and how they can be improved. The Ginibased and variance-based unfairness indices are compared in Section 5 . Sections 6 and 7 illustrate the use of the developed Gini-based fairness indices in two case studies. Section 8 introduces possible measures to reduce unfairness of the distribution of reliability between consumers. Finally, Section 9 concludes the paper.

\section{Fairness in a power system reliability context}

The perceived fairness of the distribution of reliability in power systems is determined by the fairness preferences of power system stakeholders. For example, some people want the same reliability level for everyone, while others prefer to supply a higher reliability level to people that pay more or are more in need of a higher reliability level. Distributive justice theory summarizes these two opposing fairness preferences as equity and equality [16. Equity is defined as giving everyone what they need or deserve, whereas equality is defined as treating everyone the same, regardless of differences in needs or desert [17. As both equity and equality preferences determine fairness, our proposed indices can be expressed in terms of equity or equality depending on the applied reliability indicators. In the end, the decision maker determines which reliability indicator(s) are used, preferably based on society's

\footnotetext{
${ }^{2}$ Due to the applicability of the index to different types of end-users, the general terminology 'end-users' is used in the remainder of the paper, which can refer to generators, flexibility providers or consuming entities, such as individual consumers, consumer groups or nodes.
} 
preferences ${ }^{3}$

70

\subsection{Equality and equity in a power system reliability context}

Service reliability of an electric power system is defined as the ability to adequately satisfy the demand under give operating conditions for a given time interval [18]. Reliability is commonly evaluated using reliability indicators like energy not supplied [MWh], load curtailment $[\mathrm{MW}]$, interruption cost $[€]$ or interruption frequency $[\#]$. These indicators are used to check if network operators meet certain reliability targets [8] and to determine penalties and rewards in reliability incentive schemes [19].

When focusing on cost-efficiency, security or adequacy, the absolute reliability level should be assessed using reliability indicators. When focusing on fairness, however, one should look at the relative reliability level. This is defined as the ratio of a reliability indicator $r_{j}$ and an appropriate base variable $b_{j}$ :

$$
\text { relative reliability level }=\frac{\text { reliability indicator }}{\text { base variable }}=\frac{r_{j}}{b_{j}}
$$

Table 1 summarizes a number of reliability indicators and their associated base variable. For example, energy not supplied [MWh] should be compared with total energy demand [MWh]. Applicable reliability indicators $r$ express the reliability level, in physical or economic terms, for individual endusers or different groups of end-users $4_{4}^{4}$ If all end-users have the same relative reliability level (e.g. $0.01 \%$ of their energy demand is not supplied), this could be considered to be a fair distribution of reliability.

The last column of Table 1 indicates if the distribution of the relative reliability level $\frac{r_{j}}{b_{j}}$ expresses equality or equity. A definition of fairness based on ENS, or any other physical reliability indicator, carries the notion of equality, as it does not consider individual-specific characteristics associated with need. Interruption cost, on the contrary, is obtained by multiplying ENS with its value to consumers, represented by the value of lost load (VOLL) $V_{j},\left(r_{j}=I C_{j}=E N S_{j} \cdot V_{j}\right)$. As VOLL is a measure of the consumer valuation of ENS, it is correlated with need. Due to this correlation, the definition of fairness based on IC carries the notion of equity.

However, interruption cost does not perfectly express equity, as VOLL is not a perfect measure of need. For example, poor households may be more in need of reliable electricity supply, but will

\footnotetext{
${ }^{3}$ The discussion in this section focuses on the perceived fairness of individual end-users, but similar conclusions can be drawn on substation or regional level or for consumer groups.

${ }^{4}$ Note that 'system averaged' reliability indicators (such as SAIFI or SAIDI) can not be used to assess fairness between end-consumers, consumer groups or nodes. The base variables in the definition of 'system averaged' reliability indicators represent the complete system, whereas the base variables $b_{j}$ in the definition of fairness in this paper on the contrary represent the group of end-users experiencing a certain reliability level.
} 
Table 1: Reliability indicators and their associated base variable

\begin{tabular}{lllll}
\hline Reliability indicator $r$ & Unit of $r$ & Base variable $b$ & Unit of $\frac{r}{b}$ & Equality/Equity \\
\hline Energy not supplied $^{1}$ & MWh & Energy demand $^{2}$ & $\%$ & Equality \\
Load curtailment & MW & Power demand $^{2}$ & $\%$ & Equality \\
Total interruption duration & Min & \# consumers per group & Min/cons & Equality \\
Interruption cost & $€$ & Energy demand $^{2}$ & $€ /$ MWh & Equity \\
Total cost borne by consumer & $€$ & Energy demand $^{2}$ & $€ /$ MWh & Equity \\
RES energy curtailment & MWh & Scheduled energy output & $\%$ & Equality \\
RES energy curtailment & MWh & Capacity factor & MWh & Equality \\
\hline
\end{tabular}

${ }^{1}$ Depending on whether the index is used in an ex-ante or ex-post evaluation, resp. expected energy not served (EENS) or energy not served (ENS) is used.

2 Power and energy demand corresponds to the gross, effective request for electricity of the endconsumer, i.e., the supplied load added with the curtailed load.

${ }^{3}$ The total cost borne by consumers equals the interruption cost added with the payments made to fund a compensation scheme reduced with received compensations.

typically have a lower VOLL than rich households. On the contrary, it makes sense to provide a higher reliability level to hospitals or high VOLL industry.

If curtailment of renewable energy sources (RES) is used as a reliability indicator for RES power producers, the rated power of the installation, the scheduled energy output or the capacity factor can be used as base variable. The main difference between these base variables is that the scheduled energy output and capacity factor omit unfairness due to for instance a bad location of the installation, which is implicitly included if the rated power of the installation is used as a base. The fairness index applied in [15] thus considers both the unfairness due to the location and the energy curtailment. To assess the unfairness resulting from system operation, the scheduled energy output or capacity factor are more appropriate base variables.

\subsection{Generic fairness ratio}

The relative reliability level can be used to compare the reliability level of different end-users. Using one of the reliability indicators of Table 1, a value above the average means that this consumer's reliability level is below average. A downside of the above relative reliability indicators is that units differ and that individual values are only meaningful when compared to the average. Therefore, we propose an alternative fairness ratio where both the reliability indicator and the base variable are 
normalized using the total sum of their values:

$$
\text { fairness ratio }=\rho_{j}=\text { normalized relative reliability level }=\frac{\frac{r_{j}}{\frac{\sum_{j^{\prime} \in \mathcal{J}} r_{j^{\prime}}}{b_{j}}}}{\frac{\sum_{j^{\prime} \in \mathcal{J}} b_{j^{\prime}}}{b^{\prime}}}
$$

Fair: $\rho_{j}=1 \quad \forall j \in \mathcal{J}$

That is, the fairness ratio $\rho$ of consumer $j$ expresses the share of this end-user's unreliability $r_{j}$ in the total unreliability relative to its share in the total base variable. If this ratio equals one for all end-users in the set $\mathcal{J}$ of end-users under consideration, the distribution of reliability among the end-users is considered to be fair. If the distribution is not perfectly equal, some end-users $j$ are more $\left(\rho_{j}>1\right)$ or less affected $\left(\rho_{j}<1\right):^{5}$

Both the relative reliability level and the fairness ratio make it possible to compare the reliability level of different end-users and get a sense of the unfairness of the distribution. However, to assess total unfairness in the power system, they should be combined into a single index. The next two sections will propose Gini-based and variance-based indices that combine individual fairness ratios into a single fairness index.

\section{A Gini-based fairness index in a power system reliability context}

So far the Gini index is not applied in a power system context. Nevertheless, it is the most widely used fairness index in other application contexts, such as income [20, 21, 22, 23], insurance 24], education [25], biodiversity [26]. One of the reasons for the popularity of the Gini index is that it is easy to understand how to compute it based on Lorenz curves. This section explains how the Gini index can be calculated in a power system reliability context based on Lorenz curves and it discusses its characteristics.

\subsection{Lorenz curve}

Lorenz curves represent the distribution of reliability between end-users. A Lorenz curve plots the cumulative proportion of the relative base variable $B_{k}$ with respect to the cumulative proportion of the relative unreliability $R_{k}$. The end-users are ranked according to an increasing fairness ratio. The

\footnotetext{
${ }^{5}$ The fairness ratio $\rho_{j}$ can also be derived analytically based on the analytical formulation of the Lorenz curve (see next section), as explained in Appendix A
} 
cumulative proportion of the relative base variable $B_{k}$ can be calculated as:

$$
\begin{aligned}
& B_{k}=\sum_{j=1}^{k} \frac{b_{[j]}}{\sum_{j^{\prime} \in \mathcal{J}} b_{j^{\prime}}} \forall k=1 . . J \\
& B_{0}=0 \\
& B_{J}=1
\end{aligned}
$$

$b_{[j]}$ represents the $j^{t h}$ order statistic of the base variable, which corresponds to the base variable of the end-user having the $j^{t h}$ smallest relative unreliability $\frac{r_{j}}{b_{j}}$. Where $\mathcal{J}$ is the set of end-users under analysis and $J$ the number of end-users in the set $\mathcal{J}{ }^{6}$ The cumulative proportion of relative unreliability $R_{k}$ can be calculated as:

$$
\begin{aligned}
& R_{k}=\sum_{j=1}^{k} \frac{r_{[j]}}{\sum_{j^{\prime} \in \mathcal{J}} r_{j^{\prime}}} \forall k=1 . . J \\
& R_{0}=0 \\
& R_{J}=1
\end{aligned}
$$

$r_{[j]}$ represents the $j^{t h}$ order statistic of the reliability indicator according to $\frac{r_{j}}{b_{j}}$. The fairness ratio represents the slope of the different pieces of the piecewise-linear Lorenz curve. If $\rho_{j}=1 \forall j \in \mathcal{J}$, the Lorenz curve is a straight line with coefficient of direction equal to 1 , as illustrated by the dotted line in Fig. 1. If $\rho_{j} \neq 1 \forall j \in \mathcal{J}$, the Lorenz curve will be below the line of fairness, as illustrated by the bold line in Fig. 1. The closer the Lorenz curve is to the line of fairness, the more fair the distribution of reliability.

\subsection{Gini-based fairness index based on the Lorenz curve}

The proposed Gini-based fairness index of power system reliability $U$ is defined as the ratio of the surface area between the line of fairness and the Lorenz curve (A) over the total surface area under the line of fairness $(\mathrm{A}+\mathrm{B})$ :

$$
U=\frac{A}{A+B}
$$

Surface area B can be calculated using the surface areas of the trapezoids under each of the pieces of the piecewise-linear Lorenz curve. This leads to the following formula for $U$ :

$$
U=1-\sum_{k=1}^{J}\left(B_{k}-B_{k-1}\right)\left(R_{k}+R_{k-1}\right)
$$

The end-users $j$ are ranked according to increasing fairness ratios, i.e., $\rho_{j} \leq \rho_{j+1}$. The analytical derivation of the index is discussed in Appendix B.

\footnotetext{
${ }^{6}$ The end-users can refer to generators, flexibility providers or consuming entities, such as individual end-consumers, consumer groups or geographical regions of consumers, depending on the applied reliability indicator and base variable.
} 


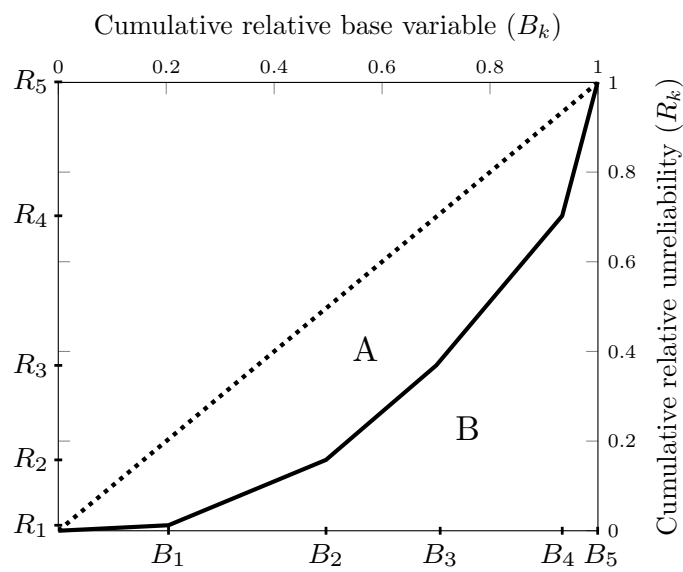

Figure 1: Lorenz curve in terms of power system reliability. The line of equality is dotted (....).

\subsection{Characteristics of Gini-based fairness indices} formulation can transform the standard deviation to a more suitable index to assess the unfairness of the distribution of reliability in power systems: the coefficient of variation. 


\subsection{Standard deviation as fairness index in a power system reliability context}

The standard deviation can be used to measure the dispersion in terms of unreliability in the system. It is a measure of the average deviation of an individual from the reference point. The standard deviation is typically defined with the mean as reference point. If we express the standard deviation in terms of the relative reliability defined in Section 2, this results in:

$$
\sigma=\sqrt{\frac{1}{J} \sum_{j=1}^{J}\left(\frac{r_{j}}{b_{j}}-E\left[\frac{r_{j}}{b_{j}}\right]\right)^{2}}
$$

Note that the reference point in this expression is the mean of the ratios $\left(E\left[\frac{r_{j}}{b_{j}}\right]\right)$, which is an average over the entities. The mean of the ratios does not have a clear physical meaning in terms of fairness, as defined in Section 2. A more suitable reference point would be the fair relative reliability level, as the ultimate objective of using the fairness index is to measure the deviations from fairness 7 Using Eq. (2), the fair relative reliability level $\left(\rho_{j}=1\right)$ equals:

$$
\frac{\sum_{j^{\prime} \in \mathcal{J}} r_{j^{\prime}}}{\sum_{j^{\prime} \in \mathcal{J}} b_{j^{\prime}}}
$$

Using this as the new reference point leads to an alternative expression of the standard deviation:

$$
\sigma_{\text {alt }}=\sqrt{\frac{1}{J} \sum_{j=1}^{J}\left(\frac{r_{j}}{b_{j}}-\frac{\sum_{j^{\prime} \in \mathcal{J}} r_{j^{\prime}}}{\sum_{j^{\prime} \in \mathcal{J}} b_{j^{\prime}}}\right)^{2}}
$$

\subsection{Characteristics of variance-based fairness indices}

Similarly to the Gini-based index, the variance-based indices do not capture where the inequality actually occurs in the distribution. However, this information can be obtained based on the fairness ratios $\rho_{j}$.

A drawback of the standard deviation is that it is hard to make comparisons between different systems with different ratios of $\frac{\sum_{j^{\prime} \in \mathcal{J}} r_{j^{\prime}}}{\sum_{j^{\prime} \in \mathcal{J}^{\prime}} b_{j^{\prime}}}$, because the standard deviation is not scale invariant [28]. This is solved by dividing the standard deviation by its reference point, leading to the coefficient of variation:

$$
C V=\frac{\sigma_{a l t}}{\frac{\sum_{j^{\prime} \in \mathcal{J}} r_{j^{\prime}}}{\sum_{j^{\prime} \in \mathcal{J}^{\prime}} b_{j^{\prime}}}}=\sqrt{\frac{1}{J} \sum_{j=1}^{J}\left(\rho_{j}-1\right)^{2}}
$$

A drawback of the coefficient of variation is that although it is expressed on a relative scale, it is not 155 defined on a zero - one scale. Therefore, it cannot be interpreted as a direct measure of the degree of unfairness.

\footnotetext{
${ }^{7}$ The fair relative reliability level can also be interpreted as the average reliability level.
} 


\section{Comparing Gini-based and variance-based fairness indices}

An extensive economic literature exists studying the properties of different unfairness indices 8 This literature focuses on both the practical use and the underlying assumptions of unfairness indices. In terms of underlying assumptions, the coefficient of variation and the Gini index are preferred over the standard deviation, because the latter implicitly assumes a quadratic welfare function, while the economic literature generally agrees on concave welfare functions 21.9

The practical use of the unfairness indices is determined by five properties, which are summarized for the four indices in Table 2 . First, except for standard deviation (12), the reference point of all use the Gini index in future academic or policy work on fairness and inequality in a power system reliability context. Therefore, the case studies below mainly focus on the Gini index, whereas the coefficient of variation has only been briefly touched upon 10

\footnotetext{
${ }^{8}$ See 29 for a comprehensive overview.

${ }^{9} \mathrm{~A}$ complete analysis of the mathematical assumptions of the considered inequality indices is beyond the scope of this paper, but in short: both the coefficient of variation and the Gini index satisfy the weak principle of transfers, symmetry, scale independence and population independence.

${ }^{10}$ Note that a large number of other inequality indices has been developed. All of them could be used to quantify inequality of power system reliability - although some have less desirable properties or require additional assumptions but none of them has such a widespread usage as the Gini index.
} 
Table 2: A summary of the practical properties of the studied indices

\begin{tabular}{|c|c|c|c|c|}
\hline & \multicolumn{3}{|c|}{ Variance-based } & \multirow[b]{2}{*}{$U$ (11) } \\
\hline & $\sigma 12$ & $\sigma_{\text {alt }} 14$ & $C V 15$ & \\
\hline Reference point has a physical interpretation & & $\checkmark$ & $\checkmark$ & $\checkmark$ \\
\hline Scale invariant & & & $\checkmark$ & $\checkmark$ \\
\hline Defined on a zero - one scale & & & & $\checkmark$ \\
\hline Transparent graphical interpretation & & & & $\checkmark$ \\
\hline Decomposable into its constituent parts & $\checkmark$ & $\checkmark$ & $\checkmark$ & \\
\hline
\end{tabular}

\section{Case study I: Electricity reliability in Norway}

Regulatory agencies can use the proposed indices to assess the level of discrimination between consumers based on detailed reliability data. This section takes a closer look at the distribution of reliability in different counties and among consumer groups in Norway. The Norwegian Water Resources and Energy Directorate (NVE) has been collecting detailed reliability data since 1995 using the FASIT tool 30. All Norwegian transmission and distribution network operators are required to report the consequences of each outage. Based on the reported interruption time period of each affected consumer type, the tool calculates the interruption duration, interrupted power, ENS and interruption cost for each consumer type at each location. The resulting reliability data are published every year for six voltage levels, 19 counties, 117 network operators and 36 consumer groups [31. ${ }^{11}$ If similar data are collected for other countries, the level of unfairness of reliability can be compared between countries. The proposed indices can be applied to any set of disaggregate reliability data, such as data on the regional level or consumer group level.

\subsection{Distribution of reliability between counties}

The distribution of unreliability among different counties is hard to evaluate by decision makers based on detailed reliability data. Fig. 2a shows the share in total ENS of each county $j$ for the 12 years. Based on this figure, one might conclude that unreliability is distributed quite equally among countries in the different years, except for some years in counties 12 and 14. However, this analysis does not take into account the relative electricity consumption of each of the counties, which is an important factor to determine the equality of the distribution of unreliability.

\footnotetext{
${ }^{11}$ See the NVE website https://www.nve.no/reguleringsmyndigheten-for-energi-rme-marked-og-monopol/ nettjenester/leveringskvalitet/leveringspalitelighet/avbruddsstatistikk/ for publications of earlier years.
} 

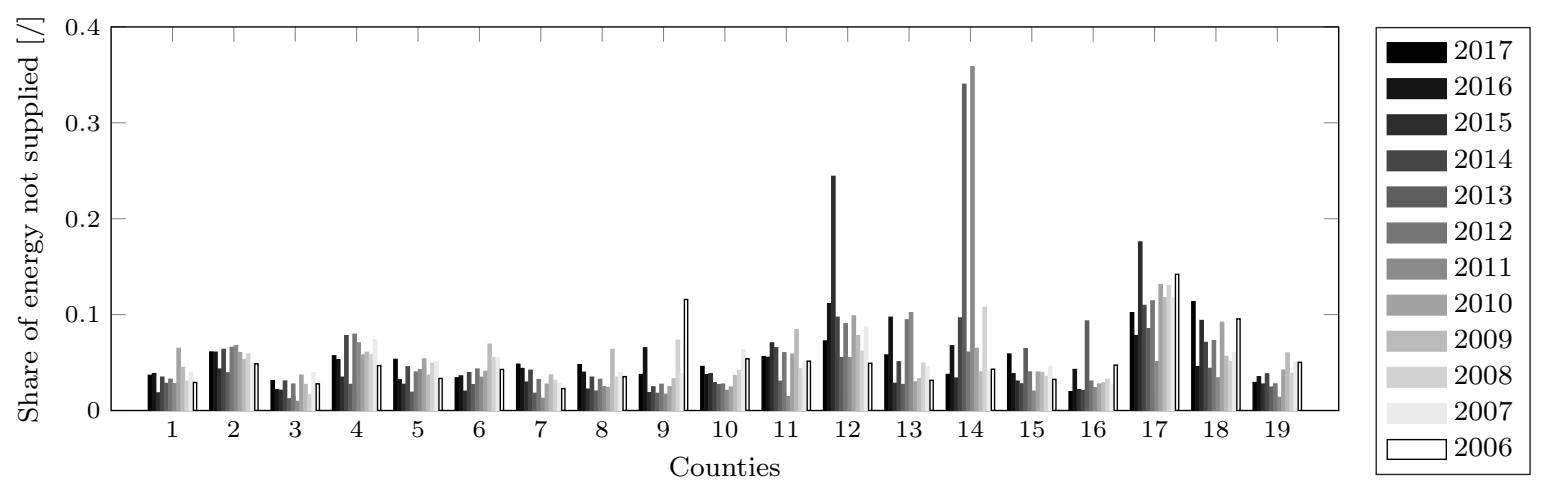

(a) Share of energy not supplied $\frac{E N S_{j}}{\sum_{j^{\prime} \in \mathcal{J}}^{E N S_{j^{\prime}}}}$

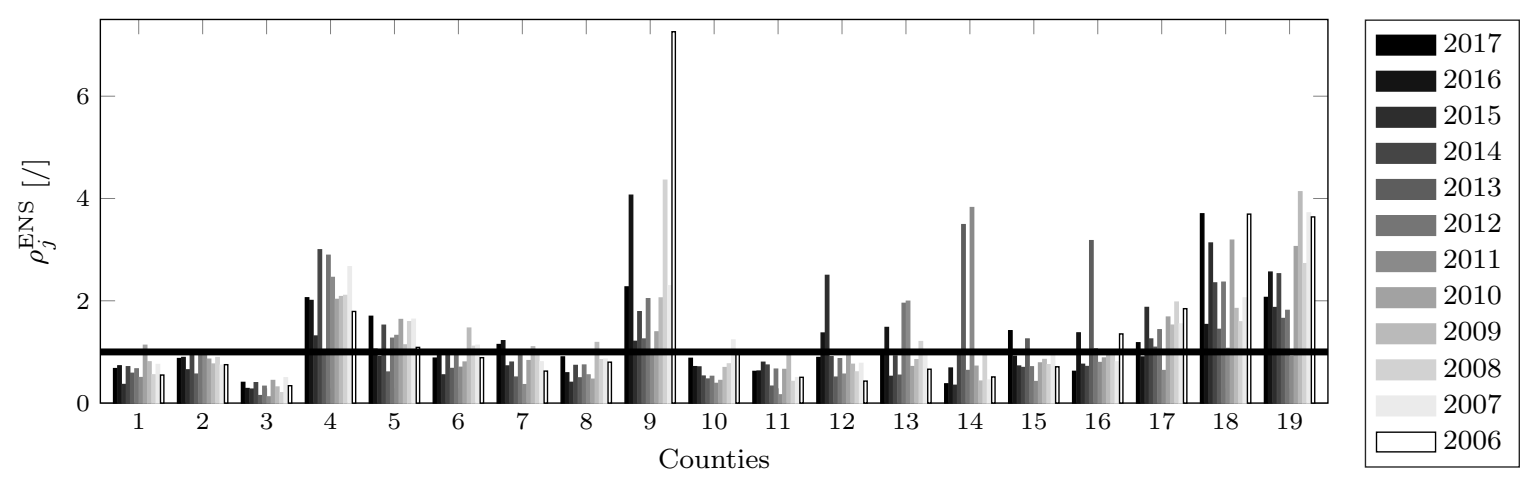

(b) Fairness ratios $\rho_{j}^{E N S}$

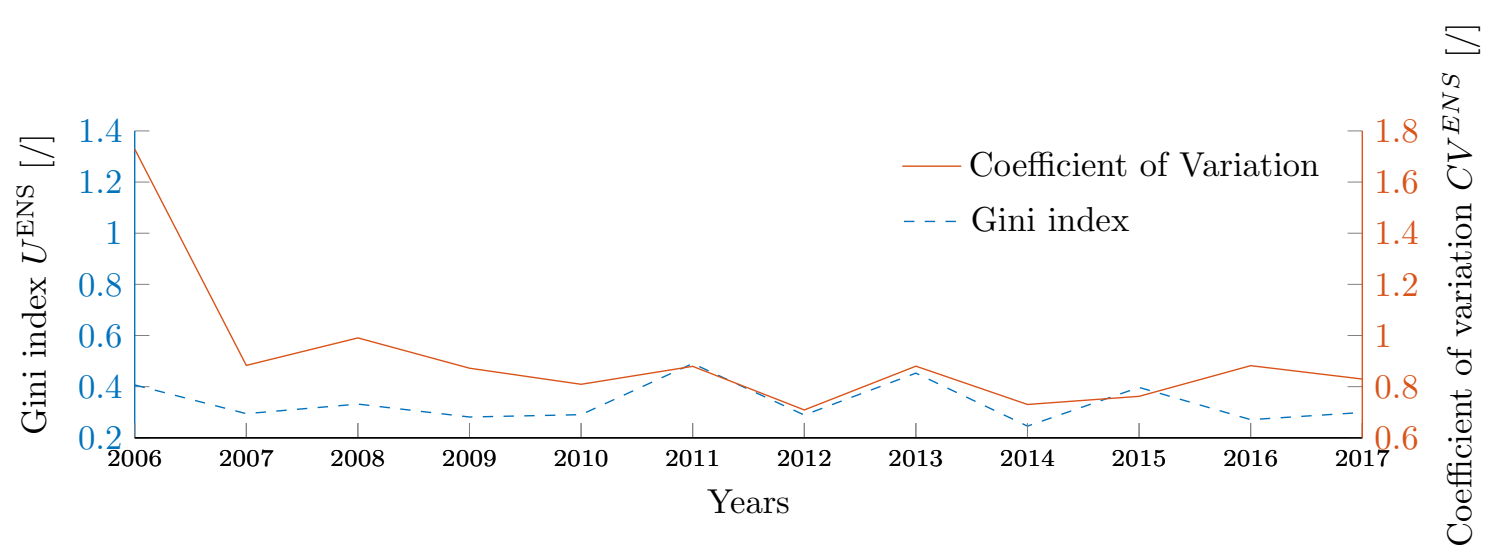

(c) Fairness index between Norwegian Counties in 2006-2016

Figure 2: Analysis of the detailed reliability data for the 19 Norwegian counties in 2006-2016 (Original data: 31]) 
Combining the data of Fig. 2a with relative electricity consumption data results in the fairness ratio $\rho_{j}^{E N S}$, which corresponds to Eq. (2) with $r_{j}$ the energy not supplied per county and $b_{j}$ the energy demand per county. Fig. $2 \mathrm{~b}$ shows that some rural areas have a fairness ratio above one, meaning that they have a relatively high level of ENS ${ }^{12}$ On the contrary, fairness ratios are below one in the more urban, southern counties, notably Oslo (county 3) and its surroundings (counties 1, 2 and 7). The high shares of energy not supplied of county 14 (Møre og Romsdal) in 2011 and 2013 was due to severe stormy weather on respectively December 24-25 and March 2. Because this county has the largest consumption of all Norwegian counties, the translation into a high fairness ratio is somewhat mitigated. Similarly, for counties with a very low consumption (like counties 4, 9, 18 and 19), blackouts with a relatively low share of energy not supplied translate into higher fairness ratios for these counties. For example, a heavy snow storm in January-February 2006 led to around 1800 MWh energy not supplied in county 9 (Aust Agder). This is only $12 \%$ of the 2006 ENS in Norway, but leads to a very large fairness ratio of 7 because it was a significant interruption for such a small county.

The reliability level differs between counties and its distribution differs over time. Evaluating the evolution of inequality in terms of reliability is only possible by aggregating the information into a single value. Fig. 2c shows the evolution of the fairness indices $U^{E N S}$ and $C V^{E N S}$ of the above distribution of reliability between counties. This figure shows that the two inequality indices generally follow the same pattern over time. Only in 2016 and 2017, the coefficient of variation and the Gini index reached opposite conclusions about the evolution of inequality. During the 12 studied years, the Gini index is between 0.24 and 0.49 , while the coefficient of variation is between 0.7 and 0.99 , except for the 2006 outlier of 1.7 ${ }^{13}$ Both indices indicate that inequality does not show a particular upward or downward trend $\sqrt{14}$, which makes sense as inequality is not a policy objective at the moment. It is also worth noting that the levels of the indices can not be compared, because the coefficient of variation is by construction not bounded between zero and one.

\subsection{Distribution of reliability between consumer groups}

The Norwegian data also make it possible to assess the unfairness of reliability between consumer groups. Fig. 3a shows the fairness ratio in terms of ENS $\rho_{g}^{\text {ENS }}$ for each consumer group $g$ for 2012 to 2016. This figure shows that agricultural and residential consumers have a fairness ratio above one,

\footnotetext{
${ }^{12}$ More specifically, the ten counties with the lowest population density are (in increasing order): 19, 13, 17, 18, 4, $5,16,15,8$ and 9 . Four of these $(4,9,18$ and 19) have a fairness ratio that is on average above 2, meaning that their relative ENS is twice as high as the national average.

${ }^{13}$ The outlier is mainly due to the high fairness index of county 9 . Note that the Gini index is less sensitive to these outliers.

${ }^{14}$ Discarding the 2006 outlier, the trendline is completely flat for the Gini index and -0.01 for the coefficient of variation.
} 
meaning that they have a relatively high level of ENS. Industry and large industry have the lowest

fairness ratio.

Similarly, Fig. 3b shows the fairness ratio $\rho_{g}^{\mathrm{IC}}$, which corresponds to Eq. (2) with $r_{j}$ the interruption cost per consumer group $g$ and $b_{j}$ the energy demand per consumer group $g$ for 2012 to 2016. This figure shows that the interruption cost of large industry is relatively low, whereas the interruption cost of commercial consumers is relatively high.

The analysis of both figures shows that large industry receives a favorable treatment, both in terms of energy not supplied and interruption cost. Although agriculture has a relatively high level of ENS, its low VOLL makes that it is on average fairly treated in terms of interruption cost. Commercial consumers are on average fairly treated in terms of ENS, but are highly unfairly treated in terms of interruption cost. This is because of their relatively high VOLL. It is worth discussing the 2013 spike in $\rho_{g}^{\text {ENS }}$ of commercial consumers. That year a severe stormy weather caused a three-day $120 \mathrm{MW}$ interruption of the Ormen Lange gasfield and pipeline 15 As the corresponding VOLL is fairly low (probably because the production and transportation facility was fairly flexible or a backup generator was available), this very long interruption translates into an average fairness ratio $\rho_{g}^{\mathrm{IC}}$.

Fig. $3 \mathrm{c}$ and Fig. $3 \mathrm{~d}$ show that in Norway there was no particular trend of unfairness in terms of reliability between consumer groups in 2012-2016, not in terms of $U^{\mathrm{ENS}}$ neither in terms of $U^{\mathrm{IC}}$. This makes sense as fairness is not a policy objective at the moment. Fig. $3 \mathrm{c}$ and Fig. $3 \mathrm{~d}$ also show that the values of the fairness indices depend on the level of consumer aggregation. The index increases if the six consumer groups, indicated with the solid line, are disaggregated into 36 groups, indicated by the dashed line.

\section{Case study II: Investment decisions}

This case study illustrates the assessment of unfairness of the distribution of service reliability among consumers in a system-development context. The impact of system reinforcements on the unfairness is assessed using a Gini-based fairness index. The case study illustrates which information can be obtained from a detailed assessment of the unfairness based on fairness indices and fairness ratios. A five-node test system, based on the Roy Billinton Reliability test system, is used.

\subsection{Data}

The base case system is shown in solid lines in Fig. 4. The existing line 4-5 has a capacity of 20 MVA. Two investments are considered in the system 16

\footnotetext{
${ }^{15}$ This is the same event that caused the 2013 spike in county 14 , as shown in Fig. $2 \mathrm{a}$

${ }^{16}$ The cost-effectiveness of the investments is not the point of concern in this analysis.
} 


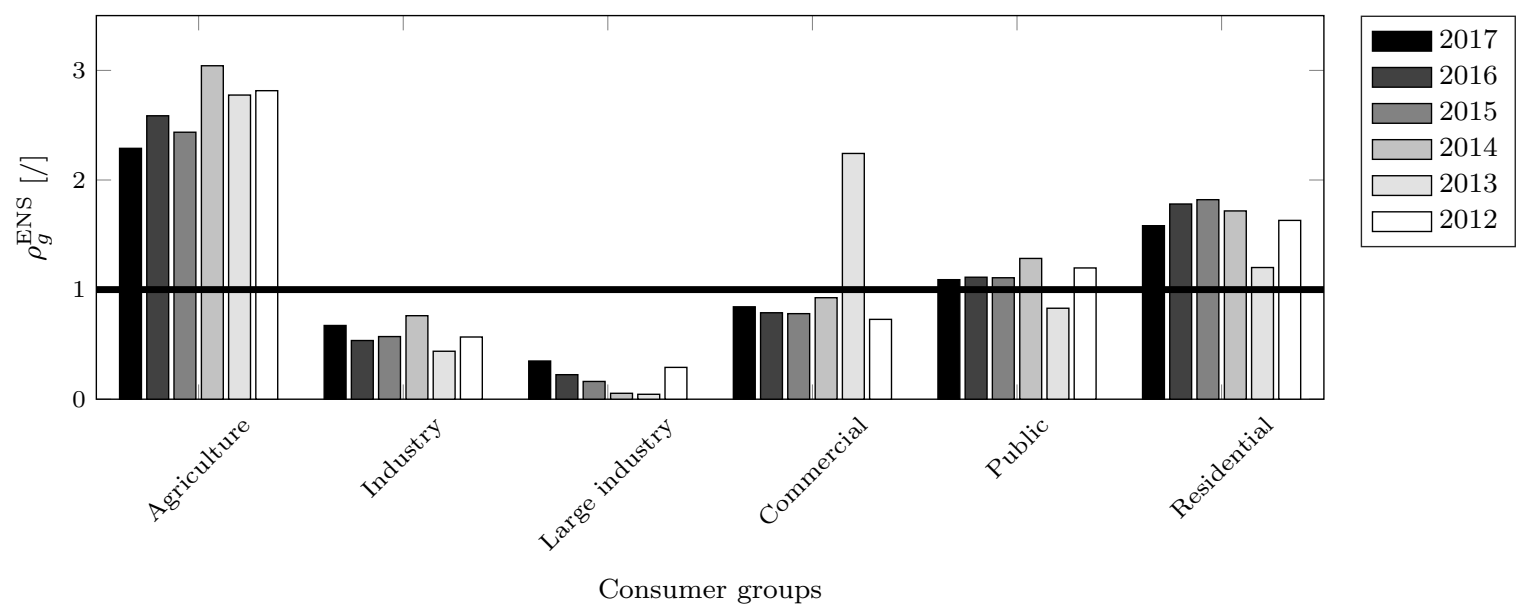

(a) Fairness ratios $\rho_{g}^{\text {ENS }}$

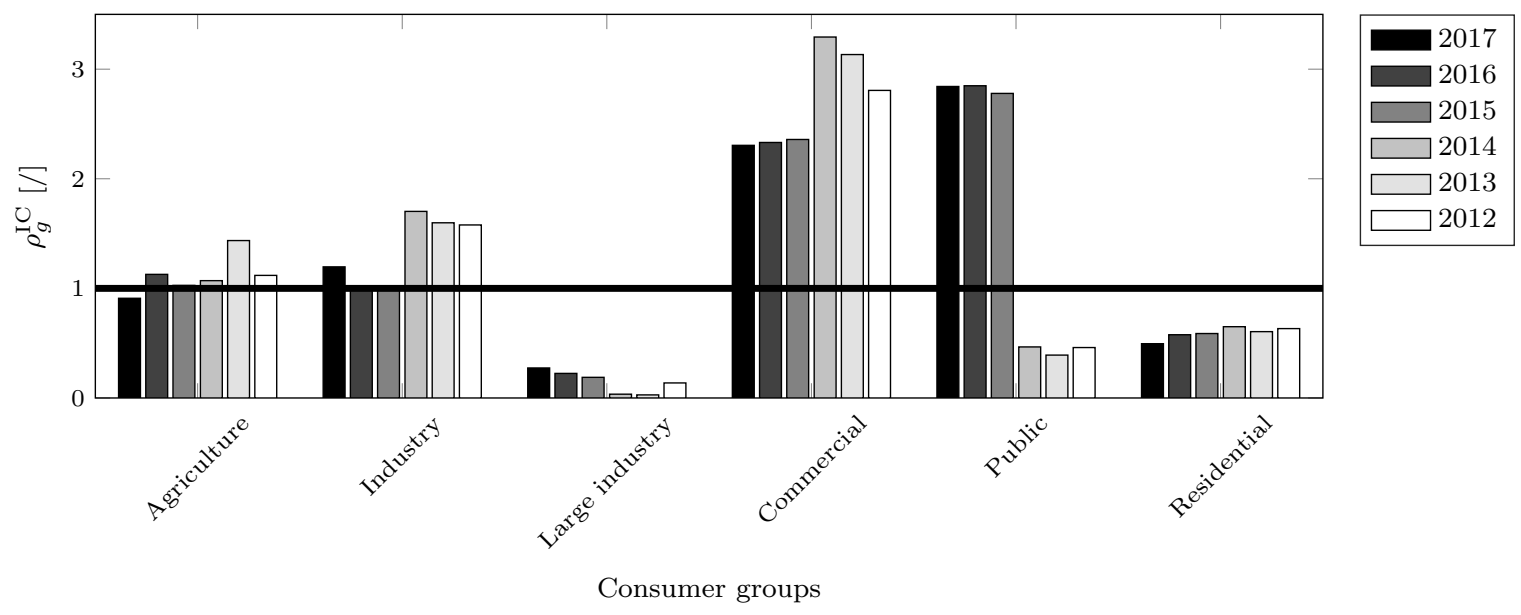

(b) Fairness ratios $\rho_{g}^{\mathrm{IC}}$

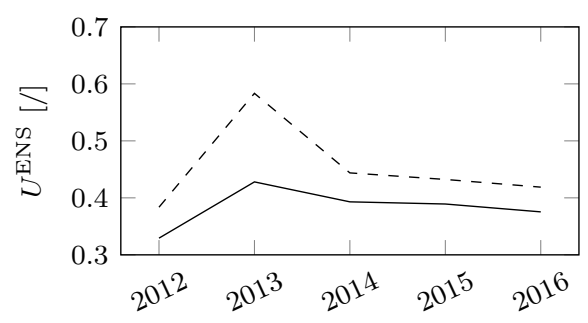

(c) $U^{\mathrm{ENS}}$

6 groups $(-)$

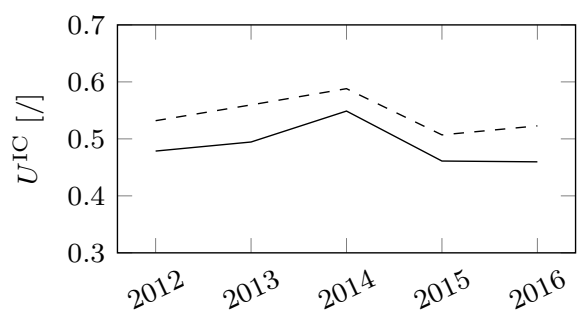

(d) $U^{\mathrm{IC}}$

36 groups (-- - -)

Figure 3: Analysis of the detailed reliability data for the different consumer groups in 2012-2016 (Original data: 31]) 
1. An additional line with capacity of 40 MVA between node 4 and 5 with phase-shifting transformer

2. Replacement of the line between node 4 and 5 with a line with 60 MVA capacity and PST

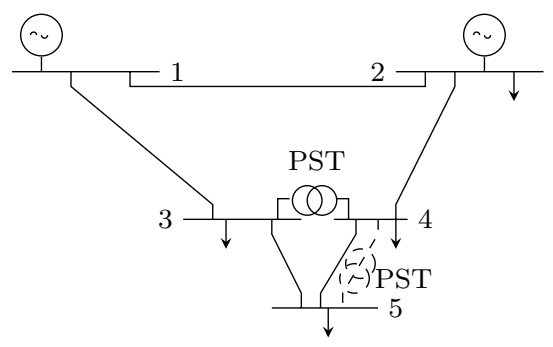

Figure 4: Circuit diagram of the test system

The base case and the two considered system reinforcements are assessed using a non-sequential, analytical state enumeration technique ${ }^{17}$ The hourly total system demand in the test system is based on the load profile defined in 33. Total system demand is distributed over two consumer groups at the different nodes, i.e., residential and non-residential consumers, as specified in [34].

Operational planning is simulated for a set of characteristic time instances that are weighted by their probability of occurrence to approximate a full year ${ }^{18}$ The selection of the time instances is specified in [34. For each time instance, in turn, a set of branch availabilities and real-time realizations of demand is considered, each with their probability of occurrence. The reliability assessment considers all N-1 branch outages and 11 demand realizations that are normally distributed, with a standard deviation of $4 \%$, around the forecast value of total demand at the corresponding time instance [35.

The simulation is executed using a MATLAB implementation interfacing with a DC securityconstrained optimal power flow (SCOPF) implemented in AMPL [3, 36. The available actions are generation redispatch, branch switching, phase-shifting transformer tap changing and load curtailment 37.

Relative load curtailment (RLC), expressed as an equivalent number of minutes, indicates the service reliability level in the system:

$$
R L C=\left(\frac{E N S}{D^{E}}\right) \cdot 8760 \cdot 60 \quad[\text { min/year }]
$$

To obtain the average service reliability level $R L C, \frac{E N S}{D^{E}}=\frac{\sum_{j \in \mathcal{J}} E N S_{j}}{\sum_{j \in \mathcal{J}} D_{j}^{E}}$, whereas the individual service reliability levels $R L C_{j}$ can be obtained if $\frac{E N S}{D^{E}}=\frac{E N S_{j}}{D_{j}^{E}}$. ENS $S_{j}$ and $D_{j}^{E}$ represent resp. the energy not supplied and the energy demand of each consumer $j$.

\footnotetext{
${ }^{17}$ Full details about the analytical state enumeration technique can be found in $\underline{32}$.

${ }^{18}$ The computation time of the power system calculations is not directly linked to the computational burden of the calculation of the unfairness indices. The calculation of the unfairness indices is a post-processing stage.
} 


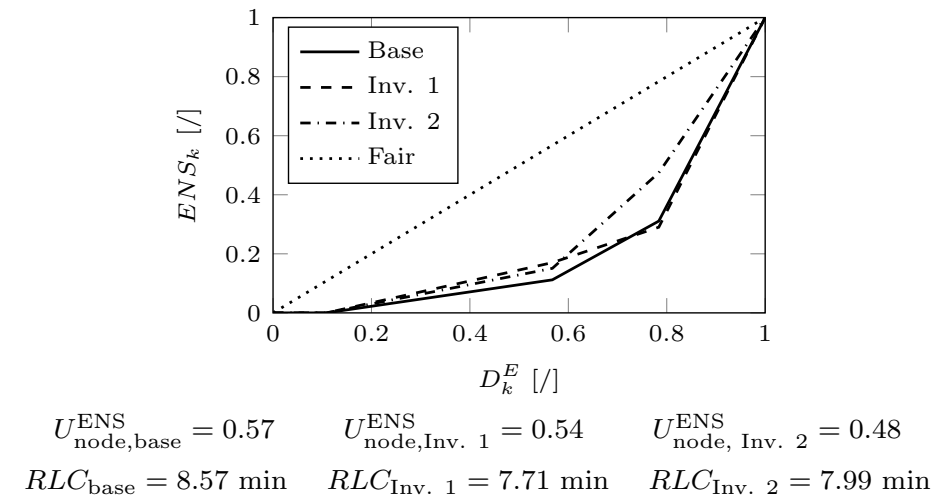

Figure 5: Lorenz curves for unfairness between nodes in terms of expected energy not served for the base case and the two investment decisions compared to the line of fairness.

The case study focuses on unfairness in terms of energy not supplied. The reliability indicator used in the unfairness index is energy not supplied $E N S_{j}$ and the base variable equals energy demand $D_{j}^{E}$ in this case. The Gini-based unfairness index in terms of energy not supplied is expressed as $U^{\mathrm{ENS}}$.

\subsection{Results}

Fig. 5 shows the Lorenz curves of unfairness between consumers at different nodes $\left(U_{\text {node }}^{\text {ENS }}\right)$ for the base case and both considered investment decisions. This figure shows that inequality is lower for both investments $\left(U_{\text {node, inv. } 1}^{\text {ENS }}=0.54\right.$ and $\left.U_{\text {node, inv. } 2}^{\text {ENS }}=0.48\right)$ compared to the base case $\left(U_{\text {node, base }}^{\text {ENS }}=0.57\right)$, as the Lorenz curves of the cases with investments 1 and 2 are closer to the line representing a fair distribution than the case without investment. Both investments reduce the relative load curtailment with nearly the same amount.

The distribution in the reduction of RLC over the different nodes is shown in Fig. 6a. Investment 1 has a slightly negative effect on node 3 , but has a positive effect on the other nodes. Investment 2 significantly reduces RLC at node 5, while having a negative impact on nodes 3 and 4 .

Fig. 6b identifies the most unfairly treated nodes by plotting the fairness ratios $\rho_{j}^{\text {ENS }}$. This figure shows that consumers from node 5 have a disproportionately low reliability level in all three cases, but the effect is reduced with investment 2 , resulting in a lower level of inequality.

On top of the inequality between nodes $\left(U_{\text {node }}^{\text {ENS }}\right)$, the index can also be calculated for inequality between different consumer groups $\left(U_{c g}^{\mathrm{ENS}}\right)$ or between different consumer groups at different nodes $\left(U^{\mathrm{ENS}}\right)$. Fairness ratios $\rho_{g}$ per group $g$, i.e. per node for $U_{\text {node }}^{\mathrm{ENS}}$ or per consumer group for $U_{c g}^{\mathrm{ENS}}$, equal:

$$
\rho_{g}^{\mathrm{ENS}}=\frac{\sum_{j^{\prime} \in I_{g}} E N S_{j^{\prime}}}{\sum_{j \in \mathcal{J}} E N S_{j}} \cdot \frac{\sum_{j \in \mathcal{J}} D_{j}^{E}}{\sum_{j^{\prime} \in I_{g}} D_{j^{\prime}}^{E}}
$$

with $I_{g}$ the set of consumers belonging to group $g$. Calculating inequality between individual consumers 


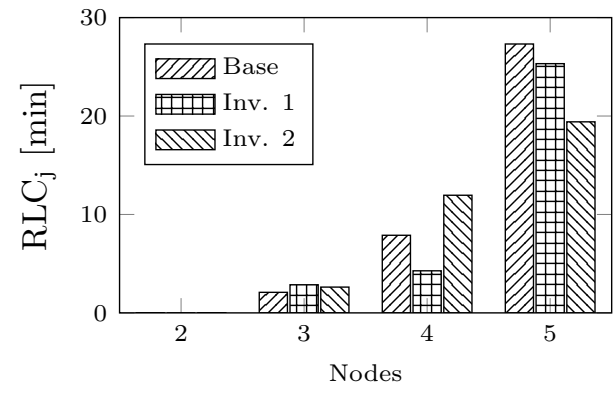

(a) Relative load curtailment per node for the base case and the two investment decisions

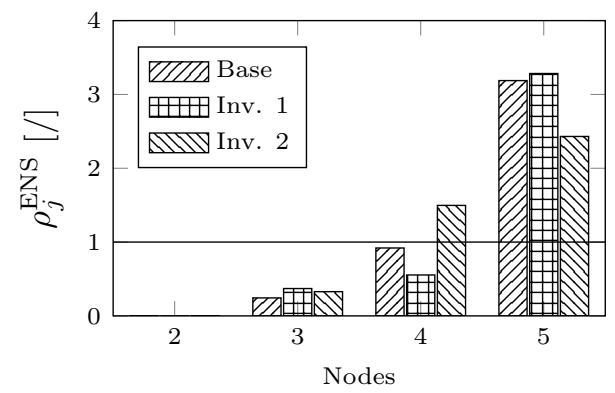

(b) Fairness ratios in terms of ENS per node for the base case and the two investment decisions

Figure 6: Distribution of unreliability among different nodes

is hard in practice, because exact energy not served and demand per consumer are not available to system operators. They only have estimations of nodal values. However, by grouping consumers per node $\left(U_{\text {node }}^{\text {ENS }}\right)$ or per consumer group $\left(U_{c g}^{\text {ENS }}\right)$, the Lorenz curve is an approximation of the Lorenz curve that considers all consumers individually. Table 3 shows that this approximation of the Lorenz curve results in lower values of the fairness indices $U_{\text {node }}^{\text {ENS }}$ and $U_{c g}^{\text {ENS }}$, quantifying the inequality between nodes and between consumer groups resp., compared to $U^{\text {ENS }}$, which considers different consumer groups at different nodes. Individual inequality is always understated if aggregation is used.

Table 3: Unfairness between nodes $U_{\text {node }}^{\text {ENS }}$, between consumer groups $U_{c g}^{\text {ENS }}$ and between different consumer groups at different nodes $U^{\mathrm{ENS}}$ for the base case and two investment decisions.

\begin{tabular}{c|ccc}
\hline & Base & Inv. 1: Additional & Inv. 2: Replacement \\
\hline$U_{\text {node }}^{\text {ENS }}$ & 0.57 & 0.54 & 0.48 \\
$U_{c g}^{\text {ENS }}$ & 0.19 & 0.34 & 0.10 \\
$U^{\text {ENS }}$ & 0.60 & 0.59 & 0.54 \\
\hline
\end{tabular}


Even if data are available at the level of individual consumers, it makes sense to calculate the inequality between nodes or between consumer groups. Consumers' perception of their peers influences data, without the use of a fairness index as proposed in this paper.

Table 4: Unfairness $U^{\text {ENS }}$ between consumers across the different nodes in the system in the two considered consumer groups for the base case and two investment decisions.

\begin{tabular}{|c|c|c|}
\hline & \multicolumn{2}{|c|}{ Consumer groups } \\
\hline & Residential & Non-residential \\
\hline Base & 0.55 & 0.47 \\
\hline Inv. 1: Additional & 0.34 & 0.64 \\
\hline Inv. 2: Replacement & 0.48 & 0.52 \\
\hline
\end{tabular}
about equality between consumer groups (e.g. residential and non-residential), the fairness index $U_{c g}^{\mathrm{ENS}}$ should be used. If they are more concerned about equality between individuals, irrespective of their consumer group, the fairness index $U^{\text {ENS }}$ should be used. Similarly, the fairness index can also be calculated within groups, such as the inequality between residential consumers or between shows that for the presented case study the inequality within the consumer groups is mostly affected by investment 1 . Inequality between residential consumers reduces, whereas inequality between nonresidential consumers increases. This also explains why inequality between consumer groups in case of investment 1 increases compared to the base case, whereas inequality between nodes or individual consumers decreases, as shown in Table 3. These conclusions are hard to make based on the original

\section{Reducing unfairness of consumers}

If the fairness index shows that the distribution of unreliability among consumers is highly unfair, measures can be taken to reduce this unfairness. The TSO can decide to safeguard the most affected consumers if load curtailment is required in the future. This requires a detailed study to see which consumers are mostly affected in a positive and negative way.

From a socio-economic perspective it might be better to have a certain level of inequality, e.g., in systems with remote and sparsely populated load points. In this case it is not economically viable to have the same level of redundancy for these remote load points as for a densely populated area. This decision might result in a higher share of energy not supplied in these remote load points. A costeffective way to reduce the level of unfairness in this case might be to invest in small, local generation 
units, possibly (partly) subsidized. Other options are a market for reliability or end-consumer contracts where the price depends on the reliability level. Bi-lateral interruptible load contracts between TSOs and large industrial consumers with flexible processes are already in place nowadays, but they might be extended to include smaller consumers as well. Although these kinds of economic compensations or reliability-based consumption choices do not affect the inequality of reliability itself, they result in a more fair distribution of the cost of unreliability. The equity between consumers will improve if consumers can indicate what they need with reliability-based electricity consumption choices. Smart grids with smart metering and demand-side management can help in this respect.

To obtain satisfactory results, the design of these measures should be done with care. Regulators should assess society's preferences in terms of the definition of fairness of the distribution of power system reliability. This assessment should include for instance end-users' perception of their peers, but also the minimal level of service reliability that should be supplied to all consumers to protect lower social classes. Based on an appropriate definition of fairness, measures can be designed to reduce the unfairness between end-users. However, the exact determination of the interruption costs is a challenge in the compensation of affected consumers and the design of reliability-based consumption frameworks. Not only are energy not served and demand per consumer hard to obtain, also exact values of lost load per node or per consumer are rarely available in practice. The level of detail in value of lost load data will improve in the future, as the fourth energy package of the European Commission prescribes that all member states have to establish at least a single estimate of VOLL for their territory and can establish a VOLL per bidding zone, if they have several ones. In many other regions such obligation does not yet exist, but more and more studies are estimating VOLL with a higher level of detail, taking into account differentiation in terms of type of consumers, time and duration. An overview of these studies can be found in 34.

\section{Conclusion}

The proposed Gini-based and variance-based fairness indices facilitate the assessment of fairness of the distribution of reliability among end-users in power systems. Their design is generic, as different reliability indicators can be used. This enables the assessment for different end-users, such as consumers, generators and flexibility providers, of two interpretations of fairness: equality and equity.

Although neither of the proposed variance-based or Gini-based indices has all ideal properties, we recommend to use the Gini index, as (i) it is the most commonly used measure of inequality in widely studied economic contexts, (ii) it has a transparent graphical presentation and interpretation based on Lorenz curves, and (iii) its bounded zero - one scale facilitates interpretation, allows for easy comparison between different reliability indicators and facilitates its use in multi-attribute decision 
[4] E. Shayesteh, J. Yu, P. Hilber, Maintenance optimization of power systems with renewable energy sources integrated, Energy 149 (2018) 577-586.

[5] Y. Tang, Q. Liu, J. Jing, Y. Yang, Z. Zou, A framework for identification of maintenance significant items in reliability centered maintenance, Energy 118 (2017) 1295-1303. 
[6] F. M. Baldursson, E. Lazarczyk, M. Ovaere, S. Proost, Cross-Border Exchange and Sharing of Generation Reserve Capacity, The Energy Journal 39 (4) (2018) 57-83.

[7] G. Perlaviciute, G. Schuitema, P. Devine-Wright, B. Ram, At the heart of a sustainable energy transition: The public acceptability of energy projects, IEEE Power and Energy Magazine 16 (1) (2018) 49-55.

[8] CEER, 6th CEER benchmarking report on quality of electricity and gas supply, Tech. rep., CEER, [Online] https://www.ceer.eu/documents/104400/-/-/ d064733a-9614-e320-a068-2086ed27be7f [Accessed: 22/09/2018] (2016).

[9] M. Wolsink, Wind power implementation: the nature of public attitudes: equity and fairness instead of backyard motives, Renewable and sustainable energy reviews 11 (6) (2007) 1188-1207.

[10] J. Cohen, K. Moeltner, J. Reichl, M. Schmidthaler, An Empirical Analysis of Local Opposition to New Transmission Lines Across the EU-27., Energy Journal 37 (3) (2016) 59-82.

[11] A. Groppi, E. Fumagalli, Network expansion by a proactive transmission system operator: A case study, Energy Policy 69 (2014) 610-623.

[12] A. Wolf, L. Wenzel, Regional diversity in the costs of electricity outages: Results for german counties, Utilities Policy 43 (2016) 195-205.

[13] H. Karimi, D. Papadaskalopoulos, G. Strbac, Integrating customers' differentiated supply valuation in distribution network planning and charging, in: European Energy Market (EEM), 2016 13th International Conference on the, IEEE, 2016, pp. 1-5.

[14] G. Strbac, D. Kirschen, R. Moreno, Reliability standards for the operation and planning of future electricity networks, Foundations and Trends in Electric Energy Systems 1 (3) (2016) 143-219.

[15] A. Latif, W. Gawlik, P. Palensky, Quantification and mitigation of unfairness in active power curtailment of rooftop photovoltaic systems using sensitivity based coordinated control, Energies 9 (6) (2016) 436-452.

[16] D. R. Forsyth, Group Dynamics, Wadsworth: Cengage Learning Belmont, 2006, Ch. Conflict, pp. $388-389$.

[17] J. Konow, Which is the fairest one of all? A positive analysis of justice theories, Journal of Economic Literature 41 (4) (2003) 1188-1239.

[18] International Electrotechnical Commission and others, Electropedia: The world's online electrotechnical vocabulary, [Online] http://www.electropedia.org/ [Accessed: 22/09/2018] (2016). 
[27] E. Heylen, M. Ovaere, S. Proost, G. Deconinck, D. Van Hertem, A multi-dimensional analysis of reliability criteria: from deterministic N-1 to a probabilistic approach, Electric Power Systems Research [Accepted for publication].

[28] F. Bourguignon, Decomposable income inequality measures, Econometrica: Journal of the Econo-

[32] E. Heylen, Evaluation of power system reliability management: Towards socially acceptable shortterm reliability criteria, Ph.D. thesis, KU Leuven (2018). 
[33] R. Billinton, S. Kumar, N. Chowdhury, K. Chu, K. Debnath, L. Goel, E. Khan, P. Kos, G. Nourbakhsh, J. Oteng-Adjei, A reliability test system for educational purposes-basic data, IEEE Transactions on Power Systems 4 (3) (1989) 1238-1244.

[34] M. Ovaere, E. Heylen, S. Proost, G. Deconinck, D. Van Hertem, How detailed value of lost load data impact power system reliability decisions: a trade-off between effciency and equity, KU Leuven Department of Economics Discussion Paper series 16.26.

[35] J. Usaola, Probabilistic load flow with correlated wind power injections, Electric Power Systems Research 80 (5) (2010) 528-536.

[36] R. Fourer, D. Gay, B. Kernighan, A modeling language for mathematical programming, Management Science 36 (1990) 519-554.

[37] T. Van Acker, D. Van Hertem, Linear representation of preventive and corrective actions in OPF models, in: Young researchers symposium, IEEE IAS/PELS/PES Benelux Chapter, 2016.

[38] M. Lubrano, The econometrics of inequality and poverty, 2017, Ch. 4: Lorenz curves, the Gini coefficient and parametric distributions, [Online:] http://www.vcharite.univ-mrs.fr/PP/ lubrano/cours/Lecture-4.pdf [Accessed: 22/09/2018].

\section{Appendix A. Analytical derivation of the fairness ratio}

Fairness in a power system reliability context is defined in terms of a relative reliability level. The cumulative distribution function of the relative unreliability $F_{E}(x)$ represents the proportion of the base variable having a relative unreliability $\frac{r_{j}}{b_{j}}$ below or equal to $x$. The cumulative distribution function of the relative unreliability corresponds to the cumulative proportion of the base variable. In our discrete case, the cumulative distribution function $\mathrm{F}_{E}$ can thus be expressed as:

$$
\mathrm{F}_{E}\left(\frac{r_{k}}{b_{k}}\right)=\sum_{j=1}^{k} \frac{b_{[j]}}{\sum_{j^{\prime} \in \mathcal{J}} b_{j^{\prime}}}=B_{k}
$$

Where $b_{[j]}$ is the $j^{t h}$ order statistic of the base variable, which corresponds to the base variable of the end-user having the $j^{\text {th }}$ smallest relative unreliability $\frac{r_{j}}{b_{j}}$.

The generic fairness ratio can be analytically derived based on the condition that the derivative of the Lorenz curve should equal one at each point for a fair distribution. The Lorenz curve is an analytical function of the inverse cumulative distribution function $\mathrm{F}_{E}^{-1}$ of the random variable under study, i.e., the relative unreliability level, and the cumulative proportion of the base variable $B[38$ :

$$
L(B)=\frac{\int_{0}^{B} \mathrm{~F}_{E}^{-1}(z) d z}{\int_{0}^{1} \mathrm{~F}_{E}^{-1}(z) d z}
$$


The Lorenz curve represents the cumulative percentage of the unreliability in the system held by a cumulative proportion of the base variable in the system. In the discrete problem at hand, the Lorenz curve in Eq. A.2 can be formulated as:

$$
L\left(B_{k}\right)=\sum_{j=1}^{k} \frac{r_{[j]}}{\sum_{j^{\prime} \in \mathcal{J}} r_{j^{\prime}}}=R_{k}
$$

If the equality or fairness condition of the Lorenz curve, i.e., $\rho_{j}=\frac{d L\left(B_{j}\right)}{d B_{j}}=1 \forall j \in \mathcal{J}$, is applied to the discrete derivative of the Lorenz curve, this results in:

$$
\begin{aligned}
& \frac{R_{j}-R_{j-1}}{B_{j}-B_{j-1}}=\frac{\frac{r_{j}}{\sum_{j^{\prime} \in \mathcal{J}^{r}} r_{j^{\prime}}}}{\frac{b_{j}}{\sum_{j^{\prime} \in \mathcal{J}^{b_{j^{\prime}}}}}}=\rho_{j} \\
& \text { Fair: } \rho_{j}=\frac{d L\left(B_{j}\right)}{d B_{j}}=\frac{\frac{r_{j}}{\sum_{j^{\prime} \in \mathcal{J}} r_{j^{\prime}}}}{\frac{b_{j}}{\sum_{j^{\prime} \in \mathcal{J}^{\prime}} b_{j^{\prime}}}}=1 \quad \forall j \in \mathcal{J}
\end{aligned}
$$

Eq. (A.4 corresponds to Eq. (2).

\section{Appendix B. Analytical derivation of the Gini-based fairness index}

The proposed Gini-based fairness index of power system reliability $U$ is defined as the ratio of the surface area between the line of fairness and the Lorenz curve (A) over the total surface area under the line of fairness $(\mathrm{A}+\mathrm{B})$. This can be analytically expressed in terms of the Lorenz curve $L(B)$ as:

$$
U=\frac{A}{A+B}=1-2 \int_{0}^{1} L(B) d B
$$

In a discrete case, this can be expressed as:

$$
U=1-2 \sum_{j=1}^{J} \int_{B_{j-1}}^{B_{j}} L(B) d B
$$

This corresponds to the integration over the piece-wise linear Lorenz curve for which the unfairness ratio represents the slope of the different pieces. Applying the trapezoidal rule for numerical integration to $(\mathrm{B} .2)$ results in:

$$
U=1-\sum_{k=1}^{J}\left(B_{k}-B_{k-1}\right)\left(R_{k}+R_{k-1}\right)
$$

which corresponds to Eq. 11 . 